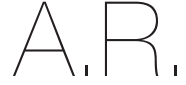

ARTIGO DE REVISÃO

Licenciatura em Ciências da Nutrição

${ }^{2}$ Centro de Reabilitação da Associação do Porto de Paralisia Cerebral,

Alameda de Cartes,192,

4300-008 Porto, Portugal

${ }^{3}$ Faculdade de Ciências da Nutrição e Alimentação da Universidade do Porto, Rua Dr. Roberto Frias, 4200-465 Porto, Portugal

"Endereço para correspondência:

Joana Sampaio

Rua das Cantoneiras, $n^{\circ} 252$,

4820-587 Quinchāes, Fafe, Braga

Portugal

joana-sampaio@live.com.pt

Histórico do artigo:

Recebido a 23 de novembro de 201 Aceite a 21 de dezembro de 2015

\title{
A IMPORTÂNCIA DA ALIMENTAÇÃO EM PARALISIA CEREBRAL
}

\author{
THE IMPORTANCE OF FEEDING IN CEREBRAL PALSY
}

Joana Sampaio ${ }^{1, *} ;$ Maria Antónia Campos²; Cláudia Afonso ${ }^{3}$

RESUMO

Uma das definições mais recentes da paralisia cerebral descreve-a como um grupo de alterações permanentes, mas não inalteráveis, do movimento e da postura, que causa limitações na atividade, as quais são atribuídas a lesões não progressivas que ocorrem no cérebro imaturo e em desenvolvimento.

Nesta população são comuns as dificuldades alimentares. Estas podem resultar de uma deficiência oro-motora (dificuldades de mastigação e/ou deglutição), disfagia e deficiência sensorial, com ou sem distúrbios comportamentais. A estas dificuldades alimentares podem, ainda, estar associados problemas de saúde como aspiração de alimentos e infeções pulmonares, refluxo gastroesofágico e obstipação. A baixa ingestão hídrica, ou até mesmo desidratação, o aumento do tempo despendido para efetuar uma refeição e a ingestão insuficiente (que pode levar a baixo peso) são outras das consequências destes problemas na alimentação. Por outro lado, existem situações em que é comum observar-se excesso ponderal por diversas razões (por exemplo, sedentarismo).

A intervenção a nível alimentar/nutricional é, por isso, fundamental para a melhoria da qualidade de vida do indivíduo. É, ainda, imprescindível que os cuidadores disponham de toda a informação e motivação para lidar com as suas especificidades, de forma a que as refeições sejam o mais agradáveis e seguras possível. Nesta população, a alimentação pode ser o nosso maior aliado.

\section{PALAVRAS-CHAVE}

Alimentação, Dificuldades e problemas alimentares, Intervenção alimentar/nutricional, Paralisia cerebral

ABSTRACT

One of the most recent definitions of cerebral palsy describes it as a group of permanent changes, but not unchangeable, of the movement and posture, causing limitations in activity, that are attributed to non-progressive damage occurring in the immature brain and under development.

In this population feeding difficulties are common. These can result from oral-motor disability (chewing and/or swallowing difficulties), dysphagia and sensory impairment, with or without behavioral disorders. These feeding difficulties may also be associated with common health problems, such as aspiration and lung infections, gastroesophageal reflux and constipation. Low water intake, or even dehydration, the increase in the time to take a meal and insufficient intake (which can lead to low weight) are other consequences of feeding problems. On other hand, there are situations where it is common overweight for various reasons (for example, sedentary lifestyle).

Dietary/nutritional intervention is essential to improve the individual's quality of life. It is also primordial that caregivers are provided with all the information and motivation to address their specificities, so that the meals are as pleasant and safe as possible. In this population, food can be our greatest ally.

\section{KEYWORDS}

Feeding, Feeding problems and difficulties, Dietary and/or nutritional intervention, Cerebral palsy

\section{INTRODUÇÃO}

A definição de paralisia cerebral (PC) foi evoluindo ao longo dos tempos e uma das definições mais recentes descreve-a como um grupo de alterações permanentes, mas não inalteráveis, do movimento e da postura, causando limitações na atividade, que são atribuídas a lesões não progressivas que ocorrem no cérebro imaturo e em desenvolvimento (1)
Associados a esta descoordenação motora estão, geralmente, distúrbios na sensação, perceção, cognição, comunicação e no comportamento, epilepsia e problemas musculares secundários (1).

A PC é uma das causas mais comuns de deficiência física na infância, nos países desenvolvidos, e afeta cerca de 2,4 nados vivos por cada 1000 (2). 


\section{A importância da alimentação em paralisia cerebral}

Quanto maior a gravidade da lesão cerebral, maiores serão as repercussões a nível alimentar, prejudicando o estado nutricional do indivíduo (3)

A intervenção a nível alimentar/nutricional é fundamental para a meIhoria da qualidade de vida do indivíduo. Perante o observado em contexto clínico no Centro de Reabilitação da Associação do Porto de Paralisia Cerebral, apresentam-se algumas das situações clínicas mais comuns nesta população, bem como as estratégias a serem implementadas, a nível alimentar e nutricional, para solucionar ou, pelo menos, atenuar estas complicações.

\section{Dificuldades de mastigação e/ou deglutição}

Na PC, devido à lesão neurológica, é muito frequente a existência de graves problemas de mastigação e deglutição de alimentos. A prevalência destas dificuldades na alimentação parece estar diretamente relacionada com a gravidade da lesão (4).

A estas dificuldades estão geralmente associados erros alimentares. Neste sentido, a população com PC parece apresentar um padrão alimentar desequilibrado, rico em açúcares e gorduras, que pode ser explicado pela maior palatibilidade destes alimentos (em caso de alimentação por via oral). Também a influência do meio envolvente e a tendência dos cuidadores para fornecer alimentos com estas características como forma de compensação alimentar são determinantes para este consumo. Parece haver, ainda, um baixo consumo diário de hortícolas, frutas e, também, de líquidos, nunca sendo este último superior a três copos por dia e feito, maioritária e erradamente, sob a forma de sumos industriais e refrigerantes (5).

A textura/consistência da alimentação destes indivíduos poderá, por vezes, ter de ser modificada, para que se adapte às dificuldades dos mesmos (6). Estes podem, e devem, consumir a mesma refeição que a população em geral. A variedade alimentar é extremamente importante na melhoria da alimentação do indivíduo com PC, no entanto, nem sempre tal acontece já que, muitas vezes, a alimentação torna-se monótona e pouco diversificada.

Os objetivos da alimentação devem centrar-se em atingir as necessidades energéticas e nutricionais e reduzir os riscos associados à ingestão alimentar, como a aspiração. Sempre que possível a via oral será a escolha primária. Caso não se consigam atingir estas necessidades com a alimentação habitual, deve, primeiramente, recorrer-se a alimentos nutritivos de alta densidade energética (6) e, em último caso, a suplementos nutricionais (7). Em casos mais graves, poder-se-á recorrer à colocação de uma sonda de alimentação.

\section{Disfagia}

A disfagia pode ser causada por uma disfunção oro-motora, anomalias anatómicas, deficiência sensorial ou distúrbios motores do esófago e, aquando da presença de refluxo gastroesofágico (RGE), pode agravar (8).

No caso da PC, esta situação é consequência de uma lesão na área do cérebro que controla a ação muscular responsável pelo correto processo de deglutição, sendo mais predominante a dificuldade em deglutir líquidos $(7,9)$ e existindo um grande risco de os alimentos serem aspirados para os pulmões, podendo causar infeções respiratórias e pneumonias recorrentes $(10,11)$. Em casos mais graves, a disfagia poderá levar a deficiências nutricionais e desidratação, resultando em perda de peso e, até, morte (9).

As manifestações clínicas da disfagia incluem, entre outros, recusa alimentar, fadiga durante a alimentação, libertação de líquidos para fora da cavidade oral, regurgitação nasal, tosse e/ou engasgamento e alteração da qualidade vocal (9)

A modificação da consistência dos líquidos, com recurso a produtos como espessantes ou água gelificada, é um dos pilares da intervenção do nutricionista em indivíduos com PC que apresentam disfagia e tem o objetivo de permitir uma ingestão hídrica segura. No entanto, a baixa aceitabilidade e adesão a líquidos com textura modificada pode contribuir para o aumento do risco de nutrição inadequada (12).

\section{Desidratação}

A desidratação é uma condição prevalente em indivíduos com PC por diversos motivos: a disfagia, a incapacidade de comunicar a sensação de sede e de beber autonomamente, na maioria dos casos, e consequente dependência da iniciativa de terceiros para lhes oferecer líquidos. Além disso, é comum oferecerem resistência à água (13). A presença de sialorreia, o ato de babar, por vezes em fio, pode também conduzir à desidratação (14).

As consequências da baixa ingestão hídrica são extremamente graves (15). Particularmente na PC, a água é essencial para o correto funcionamento intestinal, renal e hepático $(15,16)$, em situações de obstipação e em casos de polimedicação.

Algumas soluções para minimizar este problema passam por aromatizar a água com limão, laranja, cevada ou algumas gotas de groselha, optar por chás, sem açúcar e sem cafeína, ou infusões de ervas ou preparar sumos de fruta natural com adição de água. É importante não esquecer que, quando se tem sede, já o nosso organismo está desidratado, daí ser imprescindível ter a rotina de transportar diariamente uma garrafa, de forma a criar o hábito de consumo de água ao longo do dia (15), sendo possível desta forma controlar as quantidades ingeridas. Em casos de dependência é central sensibilizar os cuidadores para esta rotina. Em situações de disfagia, deve-se espessar os líquidos recorrendo a espessantes ou água gelificada (12), conforme referido anteriormente.

\section{Obstipação}

A obstipação é um problema bastante comum na PC (7) e uma das definições descreve-a como uma frequência de defecação menor que três vezes por semana ou a necessidade constante de um tratamento com laxantes (17).

A presença de hipotonicidade, incoordenação muscular esquelética e as deformidades esqueléticas agravam a obstipação (18). Para além disto, o reduzido relaxamento muscular e a imobilidade, muito comuns em pessoas com PC, dificultam o processo de defecação (18, 19). A medicação comummente utilizada nesta população, o baixo consumo de fibras alimentares e de água contribuem, igualmente, para o agravamento desta situação $(19,20)$.

A obstipação tem sido associada à redução da qualidade de vida, assim como a manifestações gastrointestinais (por exemplo, vómitos recorrentes, náuseas, agravamento do RGE, dores abdominais e saciedade precoce), choro, irritabilidade e sono inquieto (17-19). Uma alimentação rica em fibras alimentares, presentes em hortícolas, fruta crua, cereais e sementes (tais como a linhaça em pó, chia e aveia), e a elevada ingestão de água ao longo do dia são essenciais para a resolução ou minimização de problemas de obstipação (21). A utilização de laxantes pode ser necessária mas deve ser implementada, apenas e só, em última instância já que estes levam à habituação e, em algumas situações, podem ser agressivos para a flora intestinal, provocando irritações e dor que acabam por agravar este problema (17) 


\section{Refluxo Gastroesofágico}

A elevada incidência deste problema em PC está comprovada (17), sendo um dos distúrbios do trato digestivo mais prevalente (19) e superior em indivíduos que apresentam excesso ponderal (22).

Várias razões têm sido propostas para explicar tal facto, incluindo a adoção de uma posição em decúbito dorsal prolongada (o que acontece muitas vezes dado que muitos indivíduos não podem andar ou sentar-se sem apoio), o aumento da pressão intra-abdominal secundária à espasticidade, escoliose, obstipação, convulsões, alteração da consistência da dieta e medicação $(7,17,19,20)$.

Uma grande parte dos indivíduos com PC que apresentam RGE manifestam dificuldades alimentares, vómitos, recusa alimentar, infeções pulmonares recorrentes, problemas dentários, défice de crescimento e agravamento da espasticidade e dos movimentos distónicos. Se não for tratada, esta situação pode causar sintomas desagradáveis, como dor e desconforto ao deglutir os alimentos $(19,23,24)$ e, em situações mais graves, manifestar-se como esofagite (25) ou hematémese $(17,24)$.

Ultimamente tem sido demonstrado que existem alimentos que podem agravar este problema. Assim, os profissionais de saúde em geral devem centrar-se nas seguintes mudanças na alimentação para melhoria do RGE (26): privilegiar alimentos de textura suave, evitar o consumo de alimentos e bebidas muito quentes ou frias e que possam contribuir para um quadro de azia, como cafeína, bebidas gaseificadas, chocolate, frutas e sumos cítricos, alimentos ricos em gordura e muito condimentados e menta $(26,27)$.

\section{Baixo peso}

O tipo de suporte alimentar/nutricional vai depender do estado nutricional do indivíduo, da sua capacidade de ingerir quantidades adequadas de alimentos e líquidos per os e do risco de aspiração pulmonar (28). Sempre que possível, o tratamento de primeira linha deve envolver suporte nutricional oral, com o objetivo de aumentar o teor de energia, proteína e micronutrientes (geralmente, observa-se baixa ingestão, assim como baixa concentração sanguínea, de cálcio, ferro, folato, vitamina $C, D, E$, do complexo $B$, magnésio, selénio, zinco $(29,30)$ ) de alimentos e líquidos consumidos (28, 31). Para tal, deve aumentar-se o número de refeições fornecidas e a densidade energética das mesmas, limitando, ao máximo, os alimentos processados com baixo valor nutricional.

Infelizmente, a melhoria do estado nutricional em indivíduos com défice motor significativo por via oral é um desafio por si só (31). Se não se observar uma melhoria no estado nutricional após um período de tempo adequado, a suplementação deve ser considerada. Com recurso a suplementos nutricionais é possível aumentar o aporte energético e/ou proteico, bem como a ingestão de micronutrientes, ajudando, desta forma, na recuperação de peso e na melhoria do estado nutricional (28). Em PC, é necessário avaliar a presença de dificuldades alimentares no momento da escolha de nutrição entérica, quer através do uso de suplementos acrescentados aos alimentos (sob a forma de pós) ou de suplementos orais líquidos, para que a suplementação possa ser adaptada à alimentação, hábitos de vida e forma clínica do indivíduo. Se, apesar do apoio nutricional oral, o ganho de peso continuar a ser insuficiente, em situações mais graves (problemas de mastigação/deglutição acentuados, que tornam o momento da refeição demasiado prolongado e stressante, elevado risco de engasgamento e aspiração de alimentos, podendo conduzir à existência de infeções respiratórias), em que o risco de desnutrição é bastante elevado, poder-se-á equacionar a colocação de uma sonda nasogástrica ou gastrostomia endoscópica percutânea (PEG). A opção de acesso para a alimen- tação por sonda dependerá da duração prevista da alimentação e do estado clínico do utente (28). Uma vez que na maioria dos casos se tem de recorrer a um período de tempo superior de alimentação por sonda, o procedimento mais frequente é a colocação de PEG. Em caso de impossibilidade de uso de produtos de nutrição entérica (que podem ser adquiridos nas farmácias, mas que são bastante dispendiosos) na alimentação por sonda, a escolha de uma dieta líquida, devidamente adaptada às necessidades individuais, pode ser uma boa opção, existindo o cuidado de triturar devidamente todos os alimentos de modo a que apresentem uma textura suficientemente fluida para passar na sonda.

\section{Excesso de peso}

Geralmente, o excesso de peso resulta de um desequilíbrio entre a energia ingerida e a energia gasta nas atividades realizadas no dia-a-dia. É importante considerar os casos de excesso de peso, normalmente em indivíduos cuja gravidade motora da PC é menor e que são hipotónicos (32), que praticam pouco ou nenhum exercício físico mas que têm uma elevada ingestão calórica comparativamente com os gastos energéticos $(32,33)$. O crescimento atípico, as situações de compensação com alimentos de elevada densidade energética ou ingestão alimentar compulsiva são outros dos fatores responsáveis pelo excesso ponderal.

O excesso de peso poderá traduzir-se numa deficiência secundária para indivíduos com dificuldades motoras, prejudicando a coordenação e desenvolvimento muscular. Enquanto houver crescimento ósseo poderão ocorrer deformidades ósseas, em muitos casos irreversíveis (34).

No caso de pessoas com capacidade de marcha, esta poderá ser gravemente comprometida devido ao excesso de peso $(35,36)$.

A alimentação deverá ser, assim, adaptada às reais necessidades energéticas, com auxílio de um nutricionista. Habitualmente, estão também associados diversos erros alimentares (por exemplo, a já mencionada elevada ingestão de gorduras e açúcares) e, como tal, deve existir uma preocupação acrescida com a alimentação, por parte do utente ou, em caso de dependência, dos seus cuidadores. Sempre que seja possível, deve aconselhar-se a prática de exercício físico adaptado, aliada ao aconselhamento alimentar, para melhores resultados.

\section{ANÁLISE CRÍTICA}

A informação existente relativa ao papel da alimentação em PC é, ainda, escassa, sendo que maior parte dos estudos realizados tem como população base as crianças/jovens, o que pode estar também relacionado com o facto de a esperança média de vida ser baixa, embora tenha vindo a aumentar (37-39). Esta realidade exige que mais estudos sejam desenvolvidos para que se possa responder às necessidades de toda a população com PC e, assim, melhorar o seu estado de saúde e a qualidade de vida destes indivíduos e das suas famílias. Deste modo, o presente trabalho procura uniformizar, de acordo com a informação disponível e a prática clínica, as estratégias aplicadas a nível alimentar/nutricional em PC. No entanto, cada caso é um caso, dadas as imensas particularidades e variações interindividuais, não se podendo, apesar de tudo, generalizar o tipo de intervenção e assumir que a sua eficácia é idêntica em todos os indivíduos com PC.

\section{CONCLUSÕES}

A alimentação desempenha um papel decisivo na saúde de todos os indivíduos, sendo de especial importância nos que têm PC, pelas manifestações clínicas e nutricionais tão distintas e diversas que exigem uma intervenção alimentar/nutricional específica e individualizada. 
Muitas vezes, porém, e por mais adequada que seja esta intervenção, dada a gravidade da situação clínica, a mesma não se reflete numa melhoria do estado nutricional, contribuindo, no entanto, para uma diminuição dos problemas de saúde associados e, consequentemente, para a melhoria da qualidade de vida.

\section{AGRADECIMENTOS}

Centro de Reabilitação da Associação do Porto de Paralisia Cerebral, Alameda de Cartes, 192, 4300-008 Porto.

\section{REFERÊNCIAS BIBLIOGRÁFICAS}

1.Colver A, Fairhurst C, Pharoah PO. Cerebral palsy. Lancet. 2014;383(9924):1240-9 2.Oskoui M, Coutinho F, Dykeman J, Jette N, Pringsheim T. An update on the prevalence of cerebral palsy: a systematic review and meta-analysis. Dev Med Child Neurol. 2013;55(6):509-19.

3.Sullivan PB, Juszczak E, Lambert BR, Rose M, Ford-Adams ME, Johnson A. Impact of feeding problems on nutritional intake and growth: Oxford Feeding Study II. Dev Med Child Neurol. 2002;44(7):461-7.

4.Dahlseng MO, Finbraten AK, Juliusson PB, Skranes J, Andersen G, Vik T. Feeding problems, growth and nutritional status in children with cerebral palsy. Acta Paediatr. 2012;101(1):92-8

5.Lopes PA, Amancio OM, Araujo RF, Vitalle MS, Braga JA. Food pattern and nutritional status of children with cerebral palsy. Rev Paul Pediatr. 2013;31(3):344-9.

6.Zapata LFG, Mesa SLR. La alimentación del niño con parálisis cerebral un reto para el nutricionista dietista. Perspectivas desde una revisión. Perspect Nutr Humana. 2010;12:77-85.

7.Villares JM, Sanz ML, Segovia MJ, Zanuy MA. Alimentación en el paciente con parálisis cerebral. Acta Pediatr Esp. 2001;59(1):17-25.

8.Calis EA, Veugelers R, Sheppard JJ, Tibboel D, Evenhuis HM, Penning C. Dysphagia in children with severe generalized cerebral palsy and intellectual disability. Dev Med Child Neurol. 2008;50(8):625-30.

9.Marrara JL, Duca AP, Dantas RO, Trawitzki LV, Lima RA, Pereira JC. Swallowing in children with neurologic disorders: clinical and videofluoroscopic evaluations. Pro Fono. 2008;20(4):231-6.

10.Rogers B, Arvedson J, Buck G, Smart P, Msall M. Characteristics of dysphagia in children with cerebral palsy. Dysphagia. 1994;9(1):69-73.

11.Erasmus CE, van Hulst K, Rotteveel JJ, Willemsen MA, Jongerius PH. Clinical practice: swallowing problems in cerebral palsy. Eur J Pediatr. 2012;171(3):409-14.

12.Sura L, Madhavan A, Carnaby G, Crary MA. Dysphagia in the elderly: management and nutritional considerations. Clin Interv Aging. 2012;7:287-98.

13.Santos MT, Batista R, Guare RO, Leite MF, Ferreira MC, Durao MS, et al. Salivary osmolality and hydration status in children with cerebral palsy. J Oral Pathol Med. 2011;40(7):582-6

14.Walshe M, Smith M, Pennington L. Interventions for drooling in children with cerebral palsy. Cochrane Database Syst Rev. 2012;11:CD008624.

15.Hidratação adequada em meio escolar. Programa Nacional para a Promoção da Alimentação Saudável: Direção Geral da Saúde; 2014.

16. Human Hydration - Dehydration. European Hydration Institute.

17.Sullivan PB. Gastrointestinal disorders in children with neurodevelopmental disabilities. Dev Disabil Res Rev. 2008;14(2):128-36.

18.Elawad MA, Sullivan PB. Management of constipation in children with disabilities. Dev Med Child Neurol. 2001;43(12):829-32.

19.Araujo LA, Silva LR, Mendes FA. Digestive tract neural control and gastrointestinal disorders in cerebral palsy. J Pediatr (Rio J). 2012;88(6):455-64.

20.Erkin G, Culha C, Ozel S, Kirbiyik EG. Feeding and gastrointestinal problems in children with cerebral palsy. Int J Rehabil Res. 2010;33(3):218-24.

21. Wittenbrook W. Nutritional Assessment and Intervention in Cerebral Palsy. Practical Gastroenterology. 2011(92):16-32.

22.Nadaleto BF, Herbella FA, Patti MG. Gastroesophageal reflux disease in the obese: Pathophysiology and treatment. Surgery. 2015.
23.Reyes AL, Cash AJ, Green SH, Booth IW. Gastrooesophageal reflux in children with cerebral palsy. Child Care Health Dev. 1993;19(2):109-18.

24. de Veer AJ, Bos JT, Niezen-de Boer RC, Bohmer CJ, Francke AL. Symptoms of gastroesophageal reflux disease in severely mentally retarded people: a systematic review. BMC Gastroenterol. 2008;8:23.

25.Somerville H, Tzannes G, Wood J, Shun A, Hill C, Arrowsmith F, et al. Gastrointestinal and nutritional problems in severe developmental disability. Dev Med Child Neurol. 2008;50(9):712-6.

26.Vemulapalli R. Diet and lifestyle modifications in the management of gastroesophageal reflux disease. Nutr Clin Pract. 2008;23(3):293-8.

27.Kaltenbach T, Crockett S, Gerson LB. Are lifestyle measures effective in patients with gastroesophageal reflux disease? An evidence-based approach. Arch Intern Med. 2006;166(9):965-71.

28.Kuperminc MN, Gottrand F, Samson-Fang L, Arvedson J, Bell K, Craig GM, et al. Nutritional management of children with cerebral palsy: a practical guide. Eur J Clin Nutr. 2013;67 Suppl 2:S21-3.

29. Hillesund E, Skranes J, Trygg KU, Bohmer T. Micronutrient status in children with cerebral palsy. Acta Paediatr. 2007;96(8):1195-8.

30.Kuperminc MN, Stevenson RD. Growth and nutrition disorders in children with cerebral palsy. Dev Disabil Res Rev. 2008;14(2):137-46.

31.Rempel G. The importance of good nutrition in children with cerebral palsy. Phys Med Rehabil Clin N Am. 2015;26(1):39-56.

32.Bines JE. Body composition measurement: the challenge in the unwell child. Asia Pac J Clin Nutr. 1995;4(1):39-42.

33.Bansal A, Diwan S, Diwan J, Vyas N. Prevalance of obesity in children with cerebral palsy. J Clin Diagn Res. 2014;8(8):BC08-11.

34.Cao JJ. Effects of obesity on bone metabolism. J Orthop Surg Res. 2011;6:30.

35.Pataky Z, Armand S, Muller-Pinget S, Golay A, Allet L. Effects of obesity on functional capacity. Obesity (Silver Spring). 2014;22(1):56-62.

36. Hills AP, Parker AW. Locomotor characteristics of obese children. Child Care Health Dev. 1992;18(1):29-34.

37.Strauss D, Brooks J, Rosenbloom L, Shavelle R. Life expectancy in cerebral palsy: an update. Dev Med Child Neurol. 2008;50(7):487-93.

38.Strauss D, Shavelle R. Life expectancy of adults with cerebral palsy. Dev Med Child Neurol. 1998;40(6):369-75.

39.Strauss DJ, Shavelle RM, Anderson TW. Life expectancy of children with cerebral palsy. Pediatr Neurol. 1998;18(2):143-9. 